\title{
Analysis of Radar Detection Probabilities in Time Division Duplexed Systems
}

\author{
Brad W. Zarikoff \\ Hamilton Institute \\ National University of Ireland Maynooth \\ Email: Brad.Zarikoff@nuim.ie
}

\begin{abstract}
Dynamic Frequency Selection (DFS) is the technology chosen by ETSI, FCC, and Industry Canada to provide unlicensed access for broadband radios in licensed bands. It may be viewed as a basic form of cognitive radio, where broadband radios share spectrum with radar users, and is implemented by the basestation or basestation/subscriber combination. This work looks at the likelihood that a radar pulse burst can be detected in a time-division duplexed (TDD) system with radar detection restricted to the basestation. In such a system, a periodic 'quiet time' is enforced. Compared to past assumptions on the structure of the radar and radio transmission, namely that both are random, the results shown here suggest that TDD systems will find it difficult or impossible to achieve current FCC and ETSI requirements with certain frame/schedule durations. We present expressions for the number of detected pulses and simulations that demonstrate the trade-offs of varying the uplink/downlink rate and frame/schedule duration.
\end{abstract}

Index Terms-DFS, radar detection, cognitive radio, time division duplexed systems

\section{INTRODUCTION}

Recently, the Federal Communications Commission (FCC) opened the 5250 to $5350 \mathrm{MHz}$ and 5470 to $5725 \mathrm{MHz}$ bands to unlicensed broadband wireless use [1], [2]; in Europe, the 5470 to $5725 \mathrm{MHz}$ European Telecommunications Standards Institute (ETSI) band is similarly used. As these bands are traditionally used for radar purposes (primarily for weather and military uses), the FCC and ETSI have imposed certain restrictions for unlicensed broadband wireless use, and refer to these restrictions as dynamic frequency selection (DFS).

The FCC and ETSI restrictions require the unlicensed communications equipment to spend time sensing the active channel for radar presence, before and after establishing a session. During the communications session, the BS must continuously look for radar pulses, an activity referred to as 'In Service Monitoring'. In the case that a radar pulse train is detected, the basestation (BS) must organize the communications session to be relocated to another channel.

Radar waveforms are seen as pulse trains from a radio LANs (RLANs) point of view, due to rotation of the radar antenna. These periodic radar pulses inherently provide a mechanism to avoid false positives due to random interference (i.e. by using a correlator). An intelligent, implementation specific algorithm that uses the pulse widths and spacing after initial pulse identification is necessary to determine whether a radar pulse is present or not. For this work, we do not consider any

\author{
Douglas J. Leith \\ Hamilton Institute \\ National University of Ireland Maynooth \\ Email: Doug.Leith@nuim.ie
}

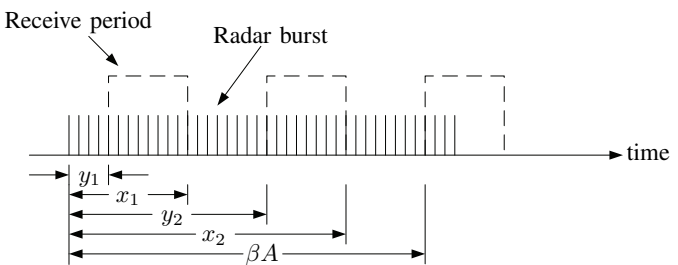

Fig. 1. An example of the signal model, with $t=2.5, T_{R}=T_{T}=5$, $T=10, \Delta=0.625, N=40$. The dashed regions correspond to the basestation receive schedule.

specific implementation, but assume that a defined number of consecutive radar pulses must be identified in order for any algorithm to be successful.

The FCC and ETSI DFS bands are not technology dependent, and may be used by frequency or time division duplexed radios. In a time-division duplexed (TDD) system, radar detection may be done with sampled data from the RF receive chain. However, one caveat in creating a DFS-compliant TDD system (e.g. DFS-compliant WiMAX) is that the receive chain must be muted during transmission. This cannot be avoided by using a secondary receive chain, since the transmitter will still drown out any signal into the secondary receiver. Hence, the DFS system must account for this inherent 'quiet time'. Our goal is then to find the probability that a number of consecutive pulses can be detected by the RLAN, given the restrictions of the TDD schedule.

Both the FCC and ETSI have specified minimum detection rates that radios must satisfy to pass certification. These rates were calculated using a rudimentary packet model with random packet sizes and rates [3] and a 50/50 split between uplink and downlink. The model does not consider the adaptivity of radio LANs (e.g. carrier sense), nor does it allow for complex scenarios of multiple competing nodes. More importantly, the certification model assumes that both the time between each radar pulse in a burst and the duration of each receive period are random.

However, the spacing between radar pulses in most of the standard radar templates used in [1], [2] are constant, where only the first arrival pulse of a radar burst is random, while in TDD the frame/schedule structure is periodic. This points to a potential model mismatch between the random model and the TDD model. As shown in this work, it is possible to determine 
the statistical overlap of a specific radar burst and the receive period of the TDD system. This provides the focus of this paper: to provide an analytical framework for determining the probability that a radar pulse is detected by a TDD radio, and thus permit a RLAN to co-exist with a radar system.

A complete model for predicting the probability of correctly detecting pulsed radar in a TDD system would require consideration for received pulse power, detection thresholds and hysteresis, analog-to-digital gain, false positives, fading channels, and antenna characteristics such as gain and beamwidth. This work presents a step in that direction, and provides an expression for the number of radar pulses that arrive during an RF receive period and analysis of the probability that a given number of radar pulses are witnessed. We also consider the aforementioned model mismatch between the DFS certification values and the TDD model. Finally, we demonstrate a simple approach that can reduce the receive mute effect, which will allow a TDD system to pass the FCC and ETSI certification criteria.

\section{BACKGROUND}

DFS radios must exceed specified minimum radar detection probabilities, e.g. an aggregated detection probability of $80 \%$ for 4 different radar burst templates, and a lower limit of $60 \%$ for each individually [1]. Thus, the very nature of DFS radio certification is probabilistic.

Since the DFS standards were introduced, studies have looked at co-existence issues of radar and wideband communication signals [4]. In [4], [5], DFS was tested using an IEEE 802.11a radio LAN (RLAN) system operating in the vicinity of a Doppler weather radar system. The conclusion was that, from the radars' perspective, the RLAN introduced additive, uncorrelated noise. The authors note that the DFS would detect the radar sooner than the RLAN would corrupt the radar, an idea also quantified in [5].

Surprisingly, the literature is largely silent on the analysis of radar detection probabilities. Some effort has been made to spur research in this direction [5]; mention was made of TDD radio operation, but no consideration to TDD operation was provided in the analysis, and a random probability of pulse detection was used. Calculation of radar detection probabilities used in the FCC and ETSI standards were initially simulated using a basic random RLAN transmission model [3]. These probabilities are also limited to a 50\% RLAN uplink ratio, as are the certification guidelines in [1], [2]. Oddly enough, once a radio is out in the field, there does not appear to be any mechanism to stop an operator from modifying the uplink ratio, thereby completely altering the DFS detection probabilities (as shown in this paper). Otherwise, the literature on DFS for radar appears restricted to detection algorithms [6] and policy review [7]. To date, there does not appear to be any work looking at the dynamics of DFS operation in a TDD system. This work presents an significant extension of the original TDD work in [8], including a more exact expression for the number of pulses landing in the receive window and suggestions for improving a DFS radar detector.

\section{System Definition}

Let $T$ denote the period of a packet schedule for a TDD system. The packet schedule is partitioned into a transmit period $\left[0, T_{T}\right]$ and a receive period $\left[T_{T}, T\right]$. For now, we restrict $T_{T}$ to be fixed. Consider a packet schedule period during which a radar transmission starts. Let $t \in[0, T]$ be a uniformly distributed continuous random variable (r.v.) that denotes the start time within the packet schedule, $N \in$ $\left\{N_{\min }, N_{\min }+1, \cdots, N_{\max }\right\}$ be a uniformly distributed discrete r.v. that denotes the number of radar pulses in the current pulse train, and $\Delta \in\left[\Delta_{\min }, \Delta_{\max }\right]$ be a uniformly distributed continuous r.v. that denotes the pulse repetition interval (PRI). Note that the variables $t, N$, and $\Delta$ are defined as uniform in the certification procedure [1], [2]. The pulse train duration, $T_{P}$, is

$$
T_{P}=\Delta(N-1) .
$$

The contribution from the pulse widths is not included in this model, since the ratio of pulse width to pulse repetition interval is quite small $(<10 \%)[8]$. Note that we allow any end time for the pulse train, and so the pulse train may extend across multiple packet schedules. Fig. 1 shows the signal model:.

\section{A. Problem Definition}

Our goal is to analyze the probability of detecting at least $n_{0}$ pulses of a radar pulse train at the receiver of a TDD DFS radio. This statistic would be useful for analysis of a system where the detector must detect $n_{0}$ periodic bursts before a radar detect interrupt is asserted. We begin by assuming that the pulse detector will detect $100 \%$ of all incoming radar pulses, provided they land during a receive period $\left[(i-1) T+T_{T}, i T\right]$, where $i=1,2,3 \ldots$ None of the pulses that land in the transmit period are counted.

We begin by defining the number of pulses that land in the receive period as $n(t, \Delta, N)$, where $t, \Delta$ and $N$ are random variables, as noted above. Our metric of interest is

$$
\begin{aligned}
P_{n}\left(n_{0}\right) & =\operatorname{Prob}\left[n \geq n_{0}\right] \\
& =\sum_{n=n_{0}}^{\infty} \int_{t, \Delta, N} p_{n}(n, t, \Delta, N) d t d \Delta d N,
\end{aligned}
$$

where $p_{n}(\cdot)$ and $P_{n}(\cdot)$ are the probability density function (pdf) and complementary cumulative distribution function of $n$, respectively. Obviously, we require $p_{n}(n, t, \Delta, N)$ for this calculation. In the next section we present a parameterised expression for $n(t, \Delta, N)$, and follow with discussion of an analytical approach for finding $p_{n}(n)$. We then move on to simulations which provide upper bounds on the number of observed arrivals. Note that $T_{R}=T-T_{T}$, the receive period duration, and $R_{R}=T_{R} / T$, the uplink ratio, play a key role in determining $P_{n}\left(n_{0}\right)$.

\section{ANALYSis}

\section{A. Parameterisation of Number of Detected Radar Pulses}

We derive a parameterised expression for the number of pulses, $n(t, \Delta, N)$, landing within the periodic TDD receive 
window. This will be used to simplify Monte Carlo simulations of pulse trains in TDD systems in Section V.

Consider a single radar pulse train. Let $t_{0}$ represent the absolute arrival time of the first pulse in the train. Then, $t=t_{0} \bmod T$ is the arrival time relative to the start of the scheduling period, where mod is the modulus function. We define $i$ as an index for the scheduling periods, starting with $i=1$ for the first one. The train spans $N_{T}$ scheduling periods, where $N_{T}=\left\lceil\frac{t+T_{P}}{T}\right\rceil$. The scheduled receive periods can be represented as a series of intervals, $\left\{\left[(i-1) T+T_{T}, i T\right]\right\}$ for $i=\left\{1,2, \cdots, N_{T}\right\}$. The expression for $n(t, \Delta, N)$ can be formed over this periodic window, given the random arrival time $t$, number of pulses $N$ and pulse interval $\Delta$. Briefly, we calculate $n(t, \Delta, N)$ in a two-stage process. First, we form an expression for the number of pulses that arrive up to the end of every schedule. Second, we form an expression for the number of pulses that arrive up to the end of every transmit period. The difference between the two terms, plus a few correction factors, is the number of pulse arrivals during the receive schedule.

Define $x_{i}(t, \Delta, N)$ as the number of pulses that arrive between the initial radar arrival time $t$ to the end of the $i^{t h}$ schedule, and $y_{i}(t, \Delta, N)$ as the number of pulses that arrive between the initial radar arrival time $t$ to the end of the transmit period of the $i^{t h}$ schedule. Then we can define the number of pulses landing in the receive interval for schedules $i \in$ $\left\{1,2, \cdots, N_{T}-1\right\}$ as $z_{i}(t, \Delta, N)=x_{i}(t, \Delta, N)-y_{i}(t, \Delta, N)$, where

$$
x_{i}(t, \Delta, N)=\left\lfloor\frac{i T-t}{\Delta}\right\rfloor+1
$$

and

$$
y_{i}(t, \Delta, N)=\left\lfloor\frac{i T-T_{R}-t}{\Delta}\right\rfloor+1 .
$$

Then, after adding in some correction factors for the last scheduling interval and for pulse trains that terminate before the end of the first receive period, the expression for $n(t, \Delta, N)$ can then be found to be

$$
n(t, \Delta, N)=\sum_{i=1}^{N_{T}-1} z_{i}(t, \Delta, N)+\beta(N-A)-B,
$$

where $\beta=u\left(\left(t+T_{P} \bmod T\right)-T_{T}\right), A=$ $u\left(t+T_{P}-T\right)\left(\left\lfloor\frac{N_{T} T-T_{R}-t}{\Delta}+1\right\rfloor\right), B=u\left(t+T_{P}-\right.$ $\left.T_{T}\right) \min \left(\alpha\left(\left\lfloor\frac{T_{T}-t}{\Delta}\right\rfloor+1\right), N\right)-u\left(N_{T}-2\right) y_{1}(t, \Delta, N)$, $\alpha=u\left(T_{T}-t\right)$, and $u(\cdot)$ is the Heaviside unit step function. Note that $N_{T}=1$ corresponds to the case when the pulse train ends during the first scheduling interval. An example for $N_{T}=3, \beta=0$, and $A=37$ is illustrated in Fig. 1 .

\section{B. Probability Distribution of $n$}

In this section, we consider calculating the pdf of $n(t, \Delta, N)$. Ideally, we would like to have an expression for the distribution of $n$ after marginalisation of $t, \Delta$ and $N$. Although we do not obtain a closed form expression for $p_{n}(n)$, it does provide some insight and potentially a starting point for future research.
1) Random Pulse Trains: Examining the three random variables that compose $n(t, \Delta, N)$, our first challenge is the discrete random variable $N$. The radar pulse train duration $T_{P}$ in (1) is dependent on $N$, and thus the pdf of $T_{P}$ has stepped intervals. To continue, we will make the assumption that $N$ is continuous and uniform. We can then take a simple transform on $\hat{N}=N-1$, so that $v=\Delta \hat{N}$, where $v$ is a continuous random variable approximating the pulse duration $T_{P}$. Analysis of this approximate pulse train duration then falls to the product of two uniform random variables. This assumption that $p_{T_{P}}(v)$ may be approximated as a continuous function stems from the fact that $p_{\Delta}(\Delta)$ is continuous. We can use the technique in [9] to find the pdf, which is shown to be piecewise with linear and log components (not shown here due to space constraints).

2) Random Arrival Time: Using the above pdf for $T_{P}$ and that the arrival time of the radar pulse from the perspective of the DFS radio is uniformly distributed, we can form an expression for $p_{n}(n)$. First, the total time overlap between the pulse train and consecutive receive windows is

$$
g(t, v)=\int_{t}^{t+v} \sum_{i=1}^{\infty}\left(u\left(\tau-(i-1) T-T_{T}\right)-u(\tau-i T)\right) d \tau .
$$

An approximation to $n$ is

$$
\hat{n}(t, \Delta, v) \approx \frac{g(t, v)}{\Delta} .
$$

Obviously, this approach is less accurate the more receive windows are overlapped by the pulse train, on account that the transmit/quiet periods are not accounted for; this approximation does not account for the additional temporal diversity. Finally, $p_{\hat{n}}(\hat{n}, t, \Delta, v)$ can be found by calculating the pdf of (6) using the uniform pdfs $p_{t}(t)$ and $p_{\Delta}(\Delta)$ and the piece-wise pdf $p_{T_{P}}(v)$ from Section IV-B1. Note that this transformation is not straightforward due to the piecewise nature of $p_{T_{P}}(v)$, the integral used to calculate $g(t, v)$ and the correlation between $v$ and $\Delta$.

To simplify, the expression in (5) can be lower bounded by considering only the first TDD schedule. This was done in the parameterised expressions in [8] and resulted in a tight approximation. Restricting $g(t, v)$ to the first receive window, we get

$$
\begin{aligned}
g(t, v)= & (T-t) u(T-t)-\left(T_{T}-t\right) u\left(T_{T}-t\right) \\
& -(T-t-v) u(T-t-v) \\
& +\left(T_{T}-t-v\right) u\left(T_{T}-t-v\right) .
\end{aligned}
$$

For the combination of small uplink ratio $R_{R}=T_{R} / T$ and radar types where $T_{P}<T$, this bound is quite tight (within $6 \%)$. However, for radar types with a larger $T_{P}$, the gap is severe, up to $100 \%$. As $R_{R}$ is increased, the bound for $T_{P}<T$ also becomes quite loose. The temporal diversity of having multiple, closely spaced observation windows becomes more evident as $T_{P}$ exceeds $T$. Since we are already relying on two other approximations, namely a continuous $N$ distribution and a simple time to PRI ratio in (6), this lower bound proves 


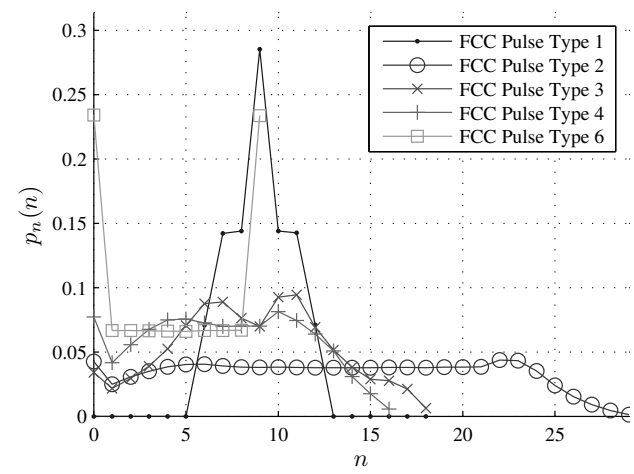

Fig. 2. Probability density function of various FCC pulse types; $10 \mathrm{~ms}$ schedule, with $R_{R}=0.5$.

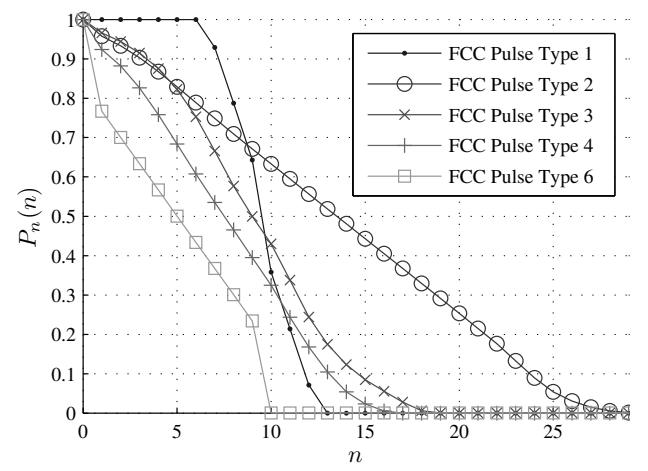

Fig. 3. Probability distribution function of various FCC pulse types; $10 \mathrm{~ms}$ schedule, with $R_{R}=0.5$.

too loose to be useful. Note that compared to the results in [8], where the lower bound considered only the number of consecutive radar pulse arrivals during a single receive period, this lower bound considers all radar pulse arrivals summed across all receive periods, which is closer to how a practical radar correlator/detector would operate. From here on, we rely on the concise parameterised expression in (4), and present simulation results based on Monte Carlo simulations for the arrival time and radar characteristics.

\section{Simulations}

In the following, we use the standard frame/schedule size of $T=10 \mathrm{~ms}$ unless otherwise stated. We focus on five different FCC specified radar types (FCC1, FCC2, FCC3, FCC4, and FCC6), as specified in [1] (FCC5 is left out as it has a different pulse train structure from the other 5 types. A summary of the parameters for each of the radar types can be found in [8], [10]. We use equation (4) in Monte Carlo simulations with $10^{5}$ runs to generate the results.

\section{A. A First Look at $p_{n}\left(n_{0}\right)$ and $P_{n}\left(n_{0}\right)$}

Fig. 2 shows the probability density $p_{n}(n)$. It is clear from the variety of these curves that deriving an expression for the marginalised pdfs is a difficult task. The associated ccdf is shown in Fig. 3, which provides a more explicit way of finding the probability of having $n_{0}$ pulses land in the receive

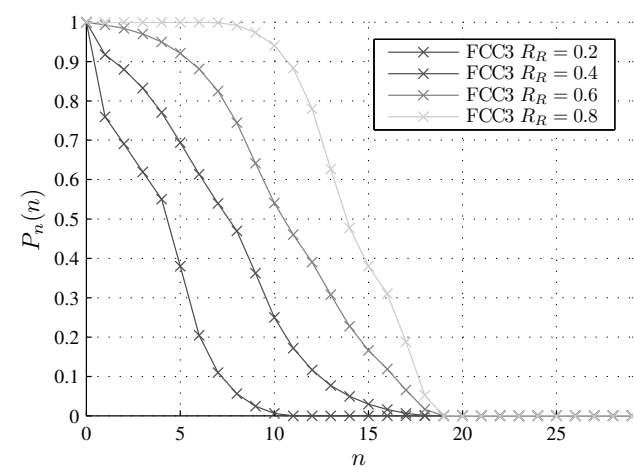

Fig. 4. Probability distribution function of FCC 3 pulse type; $10 \mathrm{~ms}$ schedule, with $R_{R}=0.2,0.4,0.6$ and 0.8 .

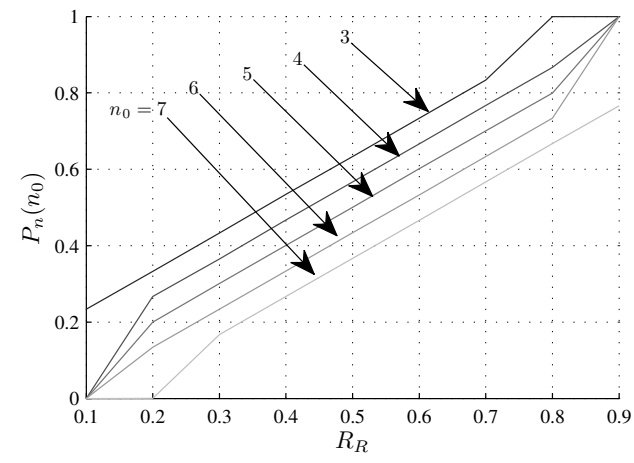

Fig. 5. $P_{n}\left(n_{0}\right)$ versus $R_{R}$ for $n_{0}=3$ to 7 , FCC pulse type $6 ; 10 \mathrm{~ms}$ schedule.

period. For example, these curves show that the probability of detecting 5 pulses of the FCC1 pulse type is $100 \%$. This owes to the fact that we account for all pulses landing in all receive periods that overlap with the interval $\left[t, t+T_{P}\right]$, where $T_{P}=24.276 \mathrm{~ms}>T$.

\section{B. Effect of Changing $R_{R}$}

In the FCC and ETSI standards, an uplink ratio of $R_{R}=$ 0.5 is used for all tests. However, once in the field, there is no restriction on what uplink ratio is used. The following shows the effect on $P_{n}\left(n_{0}\right)$ of changing $R_{R}$. Fig. 4 shows the ccdf $P_{n}(n)$ for $R_{R}=0.2,0.4,0.6$, and 0.8 for FCC3. We chose FCC3 as it provides a middle ground for the FCC pulse types. With small uplink ratio $R_{R}=0.2$, it is clear that the probability of seeing more than 5 pulses is less than $40 \%$, while if $R_{R}=0.8$, the probability of seeing more than 5 pulses is $100 \%$.

\section{Evidence of Model Mismatch}

Here we show an example where a TDD system cannot achieve the performance required for DFS certification. Fig. 5 shows the probability of detecting at least $n_{0}$ radar pulses versus the uplink ratio $R_{R}$ for FCC6. The plots use the base value of $n_{0}=3$ pulses as a satisfactory value to avoid false alarms due to noise. From the FCC memorandum 06-96 [10], it is necessary that $70 \%$ of FCC6 pulse trains must be identified. For $n_{0}=3,4$ and 5 pulses, the minimum $R_{R}$ that will satisfy 
this constraint is approximately $R_{R}=0.56,0.63$, and 0.7 , respectively. The choice of which $n_{0}$ can be supported depends on the probability of a false radar detect/miss.

These results can be compared to the random arrival model used by the ITU in [3], and discussed further in [5]. The model (briefly discussed in Section II) assumes a Bernoulli distribution with probability $p$ of detecting a single radar pulse. Of course, we need to match the parameters between the two models to provide a fair comparison. Our comparison uses the single pulse detection probability $p=0.5$ from the model in [3] to our TDD model with $R_{R}=0.5$. We do this based on the observation that as $T_{P}>>T$, the probability of detecting a randomly selected pulse in the TDD system converges to 0.5 . The TDD technique will only detect 4 or more pulses $57 \%$ of the time, while the random analysis suggests this will occur $75.4 \%$ of the time. This points out a significant mismatch between TDD and the random RLAN assumptions used to generate the FCC and ETSI criteria. The result is that system designers working with TDD systems will have to find inventive ways to pass DFS certification tests.

\section{Increasing the Probability of Detecting a Radar Burst}

In the simulations of Section V-C, it is shown that TDD systems with an uplink ratio of $R_{R}=0.5$ and a $10 \mathrm{~ms}$ schedule size are not able to meet the requirements set out by the FCC for some radar pulse types. Increasing $R_{R}$ can help meet this requirement. However, this is not acceptable for DFS certification testing as $R_{R}$ must be fixed to 0.5 , and would also reduce the downlink throughput and reduce the flexibility of the system. We suggest that the schedule duration can play a major role in increasing $P_{n}\left(n_{0}\right)$ in a system with $R_{R}=0.5$. By reducing $T$, the scheduled quiet times are reduced in length, which has the benefit of increasing the temporal aperture of the radar detector. Essentially, we are sampling the channel at a higher rate.

In TDD WiMAX systems, frame/schedule sizes can be set to $T \in\{2,2.5,4,5,8,10,12.5,20\} \mathrm{ms}$ [11]. Fig. 6 shows the results for $T=10,5$ and $2.5 \mathrm{~ms}$. Curves for FCC1 and FCC4 are shown to demonstrate the effect on the longest and shortest pulse trains $(24.276 \mathrm{~ms}$ for FCC1 and $2.4 \mathrm{~ms}$ for FCC4). For FCC4 with a small PRI, decreasing $T$ to 5 ms immediately shifts the ccdf to the right, increasing the probability of seeing 5 pulses from $68 \%$ to $93 \%$. For FCC1 with a large PRI and $T_{P}$, the shift is marginal, but does not decrease performance. For FCC6 (not shown), the probability of detecting 4 pulses is $56 \%, 63 \%, 66 \%$ and $90 \%$ for $T=10$, 5,4 and $2.5 \mathrm{~ms}$, respectively. This suggests that TDD systems must be restricted to a short schedule size in order to pass certification. Reducing the schedule size increases radar pulse detection rates, however, system designers must balance this benefit to radar detection with an increase overhead-to-payload ratio [12].

\section{CONCLUSION}

This work presents an analysis of detection of radar pulses in a time division duplexed system. We assume that dynamic

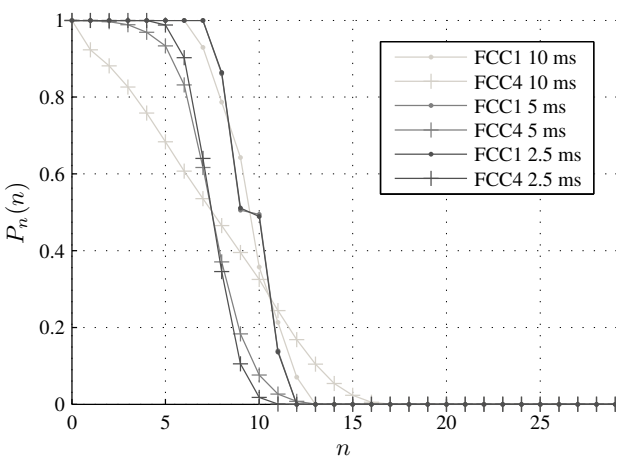

Fig. 6. $\quad P_{n}\left(n_{0}\right)$ versus $n$ for $R_{R}=0.5$, FCC pulse types 1 and 4 , and schedule size $T=10,5$, and $2.5 \mathrm{~ms}$.

frequency selection (DFS) is implemented solely at the basestation. We derive an expression for the number of pulses that arrive during the receive period of a transmission. Keeping in mind the strict requirements of the FCC and ETSI, detection probability trade-offs are investigated by varying the uplink ratio and the frame/schedule size. We find that by reducing the schedule duration from $10 \mathrm{~ms}$ to $5 \mathrm{~ms}$, the probability that 5 pulses from the shortest radar pulse burst will land in a receive window can be increased by around $25 \%$. We also detail a model mismatch between the ITU random channel model and our TDD model.

\section{REFERENCES}

[1] FCC, Title 47 CFR, Part 15 Subpart E, Federal Communications Commission, 2008.

[2] ETSI, ETSI EN 301 893, European Telecommunications Standards Institute, 2008.

[3] ITU, Recommendation ITU-R M.1652, International Telecommunications Union, 2003.

[4] A. L. Brandao, J. Sydor, and W. Brett, "5GHz RLAN Interference on Active Meteorological Radars," in IEEE 61st Veh. Tech. Conf., 2005, pp. $1328-1332$

[5] Jan Kruys, DFS Compliance Criteria, Status and Prospects, Oaktree Wireless, 2009.

[6] M. Wen and L. Hanwen, "Radar Detection for 802.11 a Systems in $5 \mathrm{GHz}$ Band," in 2005 Int. Conf. on Wireless Commun., Networking and Mobile Computing, 2005, pp. 23-26.

[7] M. J. Marcus, "Sharing Government Spectrum with Private Users: Opportunities and Challenges," IEEE Wireless Commun., vol. 16, no. 3, pp. 4-5, 2009.

[8] B. Zarikoff and D. Weldon, "Detection of Pulsed Radar in a Time Division Duplexed System," in IEEE 73rd Veh. Tech. Conf., 2011, pp. $1-6$.

[9] A. G. Glen and L. M. Leemis and J. H. Drew, "Computing the distribution of the product of two continuous random variables," Computational Statistics and Data Analysis, vol. 44, no. 1, pp. 451-464, 2004.

[10] FCC, Appendix: Compliance Measurement Procedures for UnlicensedNational Information Infrastructure Devices Operating in the 5250-5350 $\mathrm{MHz}$ and 5470-5725 MHz Bands Incorporating Dynamic Frequency Selection, Federal Communications Commission, 2006.

[11] IEEE, "IEEE Standard for Local and metropolitan area networks Part 16: Air Interface for Broadband Wireless Access Systems," IEEE Std 802.16-2009 (Revision of IEEE Std 802.16-2004), pp. C1 -2004, 2009.

[12] L. Nuaymi and N. Bouida and N. Lahbil and P. Godlewski, "Headers Overhead Estimation, Header Suppression and Header Compression in WiMAX," in IEEE 3rd Int. Conf. on Wireless and Mobile Computing, Networking and Communications (WiMOB), Oct. 2007, pp. 17-23. 